\title{
Hiperplasia suprarrenal congénita debida a deficiencia de $17 \alpha$-hidroxilasa: a propósito de una nueva mutación en el gen CYP17A1
}

\section{J.I. Perales Martíneza, B. Pina Marquésb, A. de Arriba Muñozc, E. Mayayo Dehesac, J .l. Labarta Aizpúnc y L. Loidi Fernándezd}

a Servicio de Pediatría, Hospital de Barbastro, Barbastro, Huesca, España

b Servicio de Pediatría, Hospital Universitario Miguel Servet, Zaragoza, España

c Unidad de Endocrinología Pediátrica, Servicio de Pediatría, Hospital Universitario Miguel Servet, Zaragoza,

España

d Fundación Pública Galega de Medicina Xenómica, Complejo Hospitalario Universitario de Santiago de

Compostela, Santiago de Compostela, La Coruña, España

\begin{abstract}
Resumen La enzima P450c17 cataliza 2 reacciones diferentes: 17a-hidroxilación de la progesterona y pregnenolona y segmentación de la unión del carbono 17-20 a partir de la 17,20 liasa para producir andrógenos suprarrenales. Esta enzima está codificada por el gen CYP17A1. Se presenta una paciente de 14 a nos con retraso en el desarrollo puberal y presión arterial elevada para su talla y edad. Cariotipo 46,XX. En el estudio hormonal destaca hipogonadismo hipergonadotropo, así como una insuficiencia suprarrenal y exceso mineralocorticoideo. El estudio genético mostró una mutación en homozigosis en el gen CYP17A1 (c. $753+1 G>A$ ), no descrita previamente, la cual es responsable de la fisiopatología de la deficiencia de 17ahidroxilasa. Esta entidad es una forma rara de hiperplasia suprarrenal congénita. Normalmente la enfermedad suele pasar desapercibida hasta la adolescencia o el inicio de la vida adulta y se debería sospechar ante individuos $46, X Y$ con genitales ambiguos $046, X X$ con retraso puberal que asocia hipertensión y/o hipopotasemia.
\end{abstract}

\section{PALABRAS CLAVE}

Retraso puberal; Hiperplasia suprarrenal congénita; Mineralocorticoides, Gen CYP17A1

\begin{abstract}
Congenital adrenal hyperplasia due to lack of 17 a -hydroxylase: A report of a new mutation in the gene CYP17Al

Abstract P450c17 enzyme catalyses two different reactions: the 17 a -hydroxylation of progesterone and pregnenolone, and segmenting the carbon 17-20 binding from the 17,20 lyase producing adrenal androgens. This enzyme is coded by the CYP17A1 gene. The case is presented of a 14 year old patient with delayed pubertal development and a high blood pressure for height and age. 46, XX karyotype. Hormonal studies highlighted hypergonadotropic hypogonadism, adrenal insufficiency and mineralocorticoid excess. Subsequent genetic studies showed a homozygous mutation in the CYP17A1 gene (c. $753+G>A$ ), not previously described, which is responsible for the pathophysiology of $17 \alpha$-hydroxylase deficiency. This entity is a rare form of congenital adrenal hyperplasia. The disease often goes unnoticed until adolescence or earl yadult life, and should be suspected in $46, X Y$ individuals with ambiguous genitalia or $46, X X$ with delayed puberty associated with hypertension and/ or hypokalaemia.
\end{abstract}

\section{KEYWORDS}

Delayed puberty; Congenital adrenal hyperplasia; Mineralocorticoid; CYP17A1 gene

\section{Introducción}

La deficiencia de 17a- hidroxilasa (17-OHD) es una forma rara de hiperplasia suprarrenal congénita (HSC) con una herencia autosómica recesiva y una incidencia aproximada de 1:50.000-100.000 en recién nacidosi. La enzima citocromo P450c17 está codificada por el gen CYP17, situado en el cromosoma 10q24.32. Contiene 8 exones y se expresa en la corteza adrenal y las gónadas, pero no en la placenta ni en las células de la granulosa. Las características clínicas típicas de la 17-OHD fueron descritas por primera vez en los años sesenta en una paciente con hipertensión arterial (HTA) crónica y pubertad retrasada en la que se demostraba hipopotasemia y actividad de renina y aldosterona suprimidas3. En la actualidad y desde la clonación del gen CYP17 se han descrito aproximadamente 50 mutaciones diferentes4---6. Se presenta una paciente con características clínicas y analíticas sugestivas de 17-OHD en la que se ha demostrado una mutación en el gen CYP17 todavía no descrita.

\section{Caso clínico}

Paciente mujer de 14 años con retraso en el desarrollo puberal. No presenta antecedentes familiares ni personales de interés. En la exploración física se detectan talla de $160,3 \mathrm{~cm}(-0,13 \mathrm{SDS})$ y peso de 49,3 $\mathrm{kg}(-0,57 \mathrm{SDS})$, normales, destacando ausencia de signos puberales (T1, P1, A1) con genitales externos femeninos infantiles. La edad ósea se encuentra llamativamente retrasada (9 años y 6 meses) y la presión arterial (PA) es 130/75 mmHg (p90-99 para su talla y edad), sin variaciones posturales ni asimetrías. Se realiza un estudio hormonal inicial en el que aparecen gonadotropinas muy elevadas (LH 31,92 y FSH 121,49 mUl/ml) con estradiol disminuido (<10 pg/ml), así como un exceso de ACTH (228 
$\mathrm{pg} / \mathrm{ml})$ y progesterona $(11,40 \mathrm{ng} / \mathrm{ml})$ con niveles bajos de cortisol $(0,80 \mathrm{mcg} / \mathrm{dl})$, situándonos por lo tanto en el contexto de un hipogonadismo hipergonadotropo acompa nado de insuficiencia suprarrenal. En el resto de los parámetros bioquímicos destaca únicamente un potasio en el límite bajo de la normalidad $(3,8 \mathrm{mg} / \mathrm{dl})$ con otros electrolitos normales. Se realiza un cariotipo, resultando $46, \mathrm{XX}, \mathrm{y}$ el estudio del gen SRY es negativo. La ecografía ginecológica demuestra un útero rudimentario, sin poder identificarse ovarios.

Ante los resultados obtenidos se amplía el estudio suprarrenal valorando el ritmo circadiano y se observa una pérdida del mismo con valores mantenidos de ACTH elevada y cortisol bajo en distintos momentos del día (ACTH 262 y $585 \mathrm{pg} / \mathrm{ml}$ y cortisol 0,49 y 0,10 mcg/ dl a las 8:00 y las 16:00 h, respectivamente). A continuación se analizan los distintos metabolitos intermedios de la vía del cortisol y se encuentra una elevación de pregnenolona, progesterona, desoxicorticosterona (DOCA), corticosterona y aldosterona, así como una actividad de renina plasmática suprimida. Los resultados y sus valores de referencia se presentan en la tabla 1. Finalmente se realiza estudio genético, encontrándose una mutación en homocigosis (c. $753+1 G>A$ ) en el lugar dador del splicing del exón 4 del gen CYP17A1, que no ha sido descrita en la actualidad.

En el momento del diagnóstico de presunción se inicia tratamiento con hidrocortisona vía oral 10 $\mathrm{mg} / \mathrm{m}^{2} /$ día y parches de estrógenos. Los parámetros analíticos de insuficiencia suprarrenal se han ido corrigiendo progresivamente y se normalizan las cifras de potasio y la PA. Inicia botón mamario a los 5 meses del tratamiento y mantiene un buen desarrollo puberal. A los 17 años, y tras alcanzar el desarrollo mamario completo, se añade al tratamiento gestágenos, iniciando menstruaciones regulares. La paciente presentaba llamativa osteoporosis, que ha mejorado con el tratamiento sustitutivo, pasando la Z-score de DMO en columna lumbar de -4,3 a -2,8 SDS. En la actualidad tiene 18 años, talla de 168,8 $\mathrm{cm}(0,74 \mathrm{SDS})$, peso de $65 \mathrm{~kg}(0,65 \mathrm{SDS})$, no presenta clínica sugestiva de insuficiencia suprarrenal y no han aparecido otras incidencias en la evolución.

\section{Discusión}

La síntesis de estrógenos forma parte de la esteroidogénesis que se produce en la corteza suprarrenal y que viene definida por unas reacciones enzimáticas que permiten la conversión del colesterol en diversos esteroides biológicamente activos que pertenecen a uno de los siguientes grupos principales: mineralocorticoides, glucocorticoides y andrógenos7. Como es previsible por su origen embriológico común, las células esteroidogénicas de la corteza suprarrenal y las gónadas comparten una porción significativa de la maquinaria celular responsable de la biosíntesis de esteroides y conforman en su conjunto la ruta esteroidogénica (fig. 1). La paciente presenta un defecto en la $17 \alpha$-hidroxilasa, con las características clínicas y hormonales típicas. Normalmente la enfermedad suele pasar desapercibida hasta la adolescencia o el comienzo de la vida adulta. La combinación de hipogonadismo e insuficiencia suprarrenal con el exceso mineralocorticoideo nos hacen sospechar el diagnóstico de 17-OHD. La presentación clásica es HTA, hipopotasemia y pubertad retrasada con falta de desarrollo de los caracteres sexuales secundarios en las mujeres3. En los varones pueden aparecer diferentes grados de genitales ambiguos con falta de virilización. Aproximadamente el 90\% presentan HTA o hipopotasemia en el momento del diagnóstico.

El gen CYP17 codifica la enzima P450c17, que se encarga de catalizar 2 reacciones enzimáticas: la 17 a hidroxilación de la progesterona y pregnenolona, y la segmentación de la unión del carbono 17-20 a partir de la 17,20 liasa para producir dehidroepiandrosterona y androstenediona en la glándula suprarrenal y gónadas. Alteraciones en CYP17 resultan en un deterioro de la producción de cortisol con hipersecreción secundaria de ACTH y producción de grandes cantidades de DOCA y corticosterona, que causan un estado de exceso de mineralocorticoide caracterizado por HTA, hipopotasemia y alcalosis. En consecuencia, el eje renina-angiotensina queda suprimido, provocando un defecto de síntesis de aldosterona que conlleva un hipoaldosteronismo hiporreninémico. Pese al deterioro de la producción de glucocorticoides, las concentraciones elevadas de corticosterona protegerían una posible insuficiencia suprarrenal y no aparecen síntomas clásicos de la enfermedad de Addison que ocurren en la mayoría de defectos enzimáticos suprarrenales. El tratamiento con glucocorticoides en estos pacientes corrige la HTA y la hipopotasemia, de forma que suprime el exceso mineralocorticoideo inducido por el exceso de ACTH. Por otro lado, el defecto en la producción de andrógenos suprarrenales se traduce en una falta de estrógenos y explica el hipogonadismo hipergonadotropo, de manera que aparece ausencia de vello púbico y axilar, así como falta de desarrollo mamario y amenorrea. La terapia con estrógenos inicialmente induce la pubertad en estas pacientes y se añade progesterona más adelante para regular los ciclos menstruales, previniendo de este modo también una osteoporosis en la vida adultag.

El diagnóstico hormonal de 17-OHD se basa en la elevación de DOCA, corticosterona y pregnenolona, metabolitos no disponibles en todos los laboratorios. Sin embargo, la medición de progesterona suele ser más sencilla, y en un estudio de pacientes con mutaciones en CYP17, todos ellos presentaban niveles basales elevados de 0,7 a $14 \mathrm{ng} / \mathrm{ml}$ comparados con personas normales8; por tanto, podría ser un marcador sensible de la enfermedad.

La confirmación diagnóstica se realiza por estudio molecular del gen CYP17. Desde las primeras mutaciones identificadas por Kagimoto et al.4, se han descrito más de 59 mutaciones en el gen CYP17, incluyendo simples cambios de bases, inserciones y deleciones. La localización más frecuente en la región del exón 8. La mayoría representan mutaciones esporádicas (www.hgmd.cf.ac.uk), de manera 
que 17-OHD supone aproximadamente el $1 \%$ de los casos de HSC del mundo, pero en algunos grupos étnicos aparece una prevalencia mucho más elevada y se ha sugerido un efecto fundador, como la microinserción de 4 nucleótidos en el codón 480 descritos en los descendientes menonitas canadienses10, la deleción de la fenilalanina en el codón 53 o 54 descrito en japonesesı11, una microdeleción de 9 nucleótidos cubriendo los codones 487-489 en asiáticos 12 y varias mutaciones en población brasileña5,8. En conclusión, se ha presentado una paciente mujer con una mutación en homocigosis del gen CYP17 que no ha sido descrita todavía, la cual es responsable de la fisiopatología de 17-OHD. Esta enfermedad rara deberíamos tenerla en cuenta ante individuos 46,XY con genitales ambiguos o 46,XX con retraso puberal en los que se asocia HTA y/ o hipopotasemia. La identificación de nuevas mutaciones en CYP17 es importante a la hora de comprender los mecanismos moleculares de su deficiencia y aportar información sobre la estructura enzimática de P450c17.

\section{Conflicto de intereses}

Los autores declaran no tener ningún conflicto de intereses.

\section{Bibliografía}

1. Kater C, Biglieri E. Disorders of steroid 17_hidroxylase deficiency. Endocrinol Metabol Clin North Am. 1994;23:341-57.

2. Sparkes R, Klisak I, Miller W. Regional mapping of genes encoding human steroidogenic enzymes: P450scc to 15q23-q24, adrenoxin to 11q22, adrenoxin reductase to 17q24-q25 and P450c17 to 10q24-q25. DNA Cell Biol. 1991; 10:359-65.

3. Biglieri EG, Herron MA, Brust N. 17-Hidroxylation deficiency in man. J Clin Invest. 1966;45:1946-54.

4. Kagimoto K, Waterman M, Kagimoto M, Ferreira P, Simpson E, Winter J. Identification of a common molecular basis for combined 17 $\alpha$-hidroxylase/ 17,20 lyase deficiency in two Mennonite families. Hum Genet. 1989; 82:285-6.

5. Costa-Santos M, Kater CE, Auchus RJ. Two prevalent CYP17 mutations and genotype-phenotype correlations in 24 Brazilian patients with 17_-hidroxylase deficiency. J Clin Endocrinol Metab. 2004;89:49-60.

6. Ergun-Longmire B, Auchus R, Papari-Zareei M, Tansil S, Wilson RC, New MI. Two novel mutations found in a patient with 17a -hidroxylase enzyme deficiency. J Clin Endocrinol Metab. 2006;91:4179-82.

7. Arlt W, Stewart PM. Adrenal corticosteroid biosynthesis, metabolism and action. Endocrinol Metab Clin North Am. 2005; 34:293-313.

8. Martin RM, Lin CJ, Costa EM, de Oliveira ML, Carrilho A, Villar H, et al. P450c17 deficiency in Brazilian patients: biochemical diagnosis through progesterone levels confirmed by CYP17 genotyping. J Clin Endocrinol Metab. 2003; 88:5739-46

9. Kumar Kota S, Modi K, J ha R, Narayan Mandal S. 17 a -hidroxylase deficiency: An unusual case with primary amenorrea and hypertension. Indian J Endocrinol Metab. 2011;15:127-9.

10. Imai T, Yanase T, Waterman M, Simpson E, Pratt J. Canadian Mennonites and individuals residing in the Friesland region of the Netherlands share the same molecular basis of 17 -hidroxylase deficiency. Hum Genet. 1992;89:95-6.

11. Miura K, Yasuda K, Yanase T, Yamakita N, Sasano H, Nawata H, et al. Mutation of cytochrome P-45017_ gene (CYP17) in a Japanese patient previously reported as having glucocorticoidresponsive hyperaldosteronism: With a review of J apanese patients with mutations of CYP17. J Clin Endocrinol Metab. 1996;81:3797-801.

12. Qiao J, Hu RM, Peng YD, Song HD, Peng YW, Gao GF, et al. A complex heterozygous mutation of His373Leu and Asp487- Ser488-Phe489 deletion in human cytochrome P450c17 causes 17_-hidroxylase/ 17,20 lyase deficiency in three Chinese sisters. Mol Cell Endocrinol. 2003;201:189-95. 
Tabla 1 Estudio hormonal de la paciente y los valores de normalidad

\begin{tabular}{lll}
\hline & Paciente & Valor normal \\
\hline Estradiol & $<10 \mathrm{pg} / \mathrm{ml}$ & $>10$ en inicio pubertad \\
LH & $31,92 \mathrm{mUl} / \mathrm{ml}$ & $2-15$ fase folicular \\
FSH & $121,49 \mathrm{mUl} / \mathrm{ml}$ & $3-20$ fase folicular \\
ACTH & $228 \mathrm{pg} / \mathrm{ml}$ & $8-42$ \\
Cortisol & $0,80 \mu \mathrm{g} / \mathrm{dl}$ & $5-38$ \\
Pregnenolona & $18,47 \mathrm{ng} / \mathrm{ml}$ & $0,14-2,20$ \\
Progesterona & $13 \mathrm{ng} / \mathrm{ml}$ & $0,31-1,52$ \\
DOCA & $130,9 \mathrm{ng} / \mathrm{dl}$ & $2,0-15,0$ \\
Corticosterona & $10,0 \mu \mathrm{g} / \mathrm{dl}$ & $0,10-2,0$ \\
17-OHP & $0,73 \mathrm{ng} / \mathrm{ml}$ & $0,2-0,9$ \\
11-DOC & $1,05 \mathrm{ng} / \mathrm{ml}$ & $0-8$ \\
Aldosterona & $387,7 \mathrm{pg} / \mathrm{ml}$ & $25,0-315,0$ \\
ARP & $0,50 \mu \mathrm{Ul} / \mathrm{ml}$ & $2,80-39,90$ \\
Potasio & $3,8 \mathrm{mg} / \mathrm{dl}$ & $3,5-5,1$ \\
\hline
\end{tabular}

ACTH: hormona adrenocorticotropa; ARP: actividad de renina plasmática; DOCA: desoxicorticosterona; FSH: hormona foliculoestimulante; LH: hormona luteinizante; 17-OHP: $17-$ hidroxiprogesterona; 11-DOC: desoxicortisol. 


\section{Síntesis de los esteroides}

Colesterol (Superficie externa mitocondria)

$$
\downarrow \text { Star }
$$

Colesterol (Superficie interna mitocondria) CYP11A1 $\downarrow$ 20,22-desmolasa

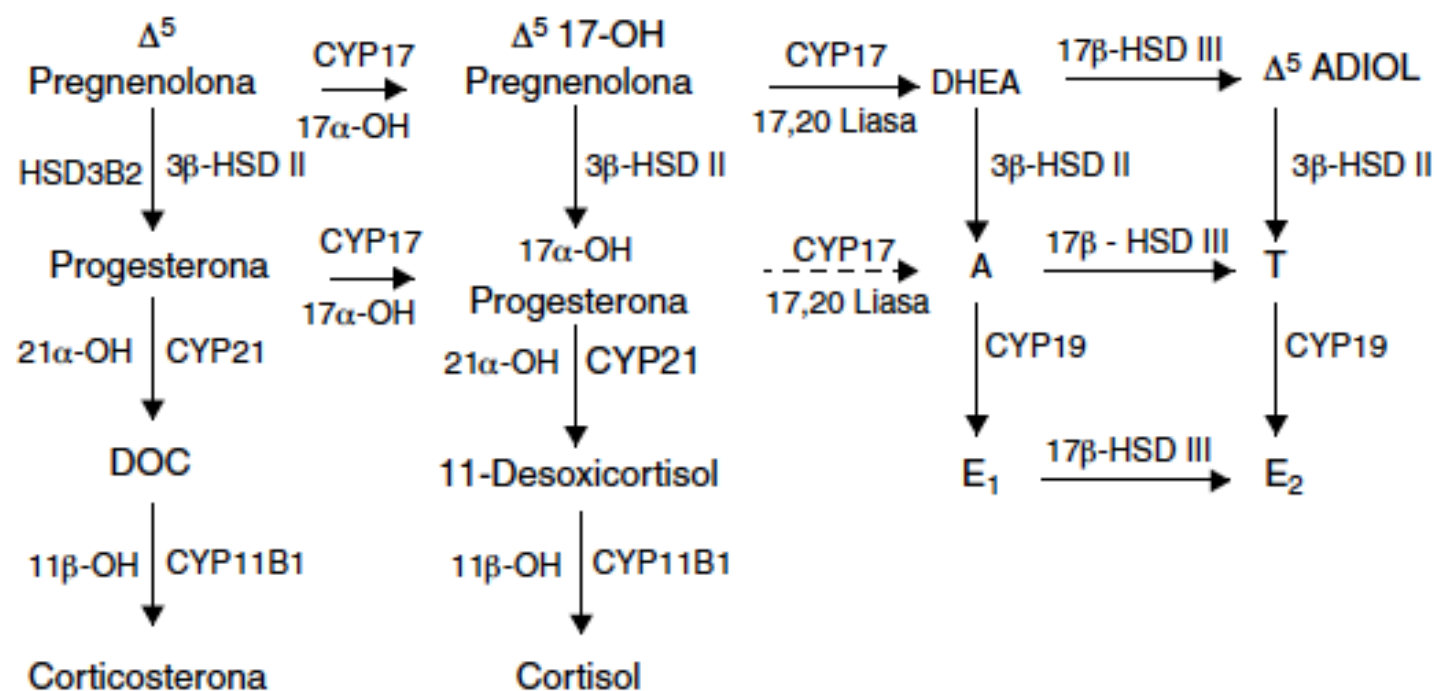
$\downarrow$ (18-OH) CYP11B2
$18-\mathrm{OH}$

Corticosterona

$\downarrow$ (18-Oxidasa) CYP11B2

Aldosterona

Mineralocorticoides Glucocorticoides Sexoesteroides

Figura 1 Ruta de la esteroidogénesis con sus enzimas, metabolitos y genes. 